\title{
JAM: an event generator for high energy nuclear collisions
}

\author{
Yasushi $\mathrm{Nara}^{1, *}$ \\ ${ }^{1}$ Akita International University, Yuwa, Akita-city 010-1292, Japan
}

\begin{abstract}
We review recent developments of an event generator JAM microscopic transport model to simulate high energy nuclear collisions, especially at high baryon density regions. Recent developments focus on the collective effects: implementation of nuclear potentials, equation of state (EoS) modified collision term, and dynamical integration of fluid dynamics. With these extensions, we can discuss the EoS dependence of the transverse collective flows.
\end{abstract}

\section{Introduction}

The transport theoretical description of nuclear collisions is necessary to understand the collision dynamics, and extract information about the properties of hot and dense matter produced in heavy-ion collisions. A correct description of non-equilibrium process in the early stages of the collisions particularly is very important. A microscopic transport model JAM has been developed for the purpose of simulating high energy nuclear collisions [1] in a similar way as in the RQMD [2] and UrQMD models [3]. In the original version of JAM, a nuclear collision is described by the so-called cascade model in which nuclear collisions are modeled by the superposition of independent binary collisions among hadrons including produced ones with a probability given by the free hadronhadron scattering cross section. In between binary collisions, hadrons follow straight line trajectories. Strings or hadron resonances may be produced at each inelastic hadron-hadron collision, and secondary products from the decay of strings or hadron resonances can scatter again, which are the main source of collective flows in the cascade model. It is well known, however, that pressure generated in the cascade type description is generally low, and fails to reproduce the collective transverse flows, such as directed or elliptic flow [4, 5]. Many body effects (multiparticle interactions), are implemented in the BoltzmannUehling-Uhlenbeck (BUU) [6] and the quantum molecular dynamics (QMD) models [7] in which nuclear meanfield potentials are introduced in the cascade model. In JAM, a nuclear mean-field is implemented $[8,9]$ based on the simplified version of the relativistic quantum molecular dynamics (RQMD/S) approach [10].

Another possible way to change the pressure of the system is to modify scattering style in the two-body collisions; selecting repulsive orbit enhances the pressure, while attractive orbit reduces the pressure [11-13]. We have demonstrated that by imposing an additional condition in the two-body scattering [14], it is possible to con-

\footnotetext{
*e-mail: nara@aiu.ac.jp
}

trol the pressure of the system and to simulate an equation of state (EoS) such as first-order phase transition or crossover transition [15-20].

Hydrodynamical description is a standard way to incorporate the effects of EoS into the dynamics. Recently, dynamical initialization of fluid has been developed $[21,22]$, in which particles are dynamically converted into fluid elements through the source terms of the hydrodynamical equations. This idea has been incorporated in JAM (JAM + hydro) in order to simulate heavy-ion collisions at high baryon density regions [23].

\section{Model}

In JAM, the nuclear mean-field is implemented based on the RQMD/S $[8,10]$ model. In this approach, the Hamiltonian is given by the sum of the single particle energy:

$$
H=\sum_{i=1}^{N} \sqrt{\boldsymbol{p}_{i}^{2}+m_{i}^{2}+2 m_{i} V_{i}}
$$

and the equations of motion

$$
\frac{d \boldsymbol{r}_{i}}{d t}=\frac{\boldsymbol{p}_{i}}{p_{i}^{0}}+\sum_{j=1}^{N} \frac{m_{j}}{p_{j}^{0}} \frac{\partial V_{j}}{\partial \boldsymbol{p}_{j}}, \quad \frac{d \boldsymbol{p}_{i}}{d t}=-\sum_{j=1}^{N} \frac{m_{j}}{p_{j}^{0}} \frac{\partial V_{j}}{\partial \boldsymbol{r}_{j}}
$$

are numerically solved. We note that the relative distance and momentum in the two-body center-of-mass frame are used for the argument of potentials. The Skyrme-type density dependent and Lorentzian-type momentum dependent potentials for baryons are included:

$$
V_{i}=\frac{\alpha}{2}\left(\frac{\rho_{i}}{\rho_{0}}\right)+\frac{\beta}{1+\gamma}\left(\frac{\rho_{i}}{\rho_{0}}\right)^{\gamma}+\sum_{k=1}^{2} \frac{C_{k}}{2 \rho_{0}} \sum_{j(\neq i)} \frac{\rho_{i j}}{1+\left(\boldsymbol{p}_{i j} / \mu_{k}\right)^{2}},
$$

where $\rho_{i}$ is a convolution of the Gaussian wave packet $\rho_{i j}$, and $\rho_{0}=0.168 \mathrm{fm}^{-3}$ is the normal nuclear density. The values of the potential parameters $\alpha, \beta, \gamma, \mu_{k}$, and $C_{k}$ $(k=1,2)$ can be found in Ref. [18] which are determined 


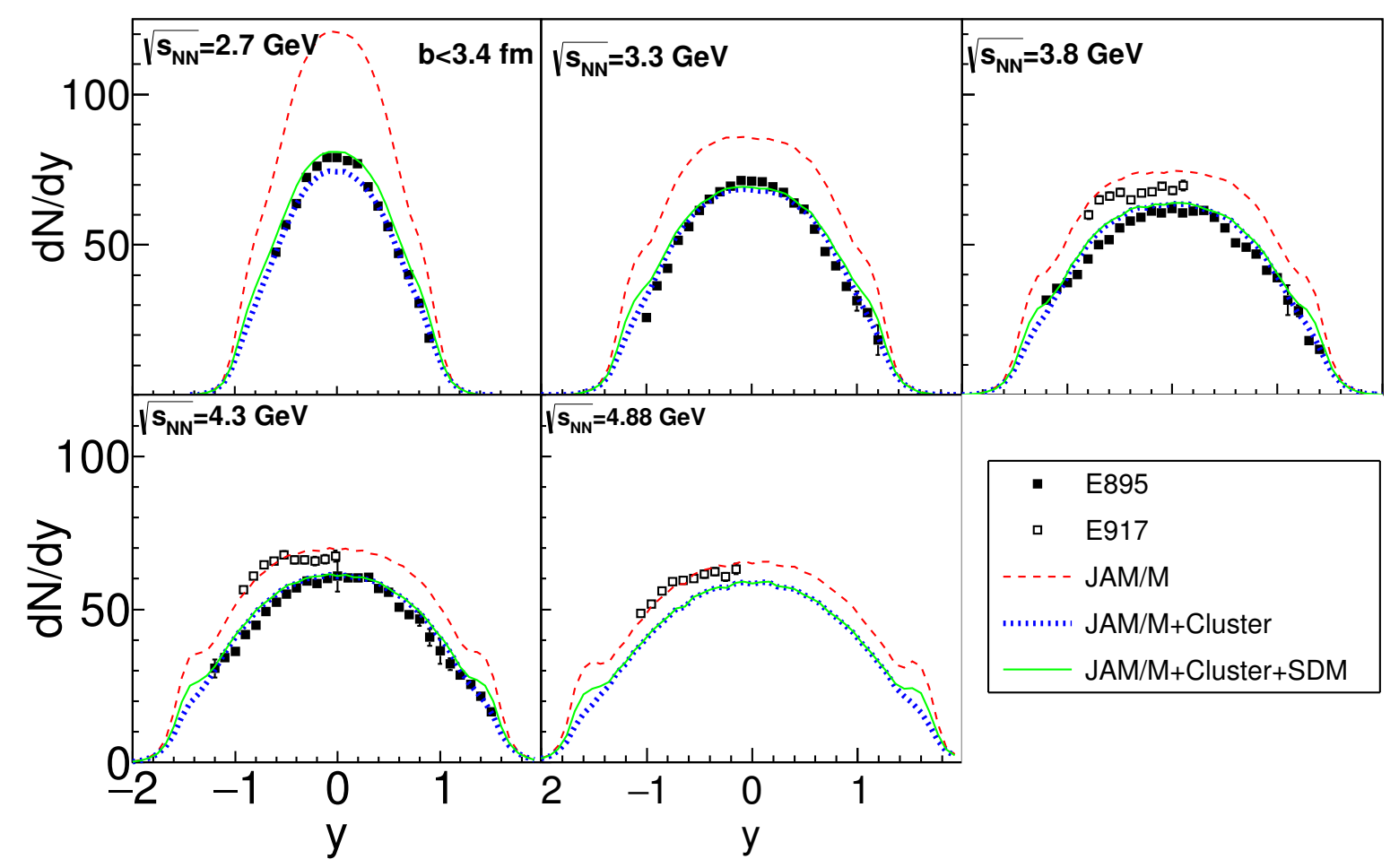

Figure 1. Rapidity distributions for protons in central $\mathrm{Au}+\mathrm{Au}$ collisions at AGS energies are compared with the JAM mean-field mode (JAM/M) (dashed lines), and with nuclear cluster formation (JAM/M+Cluster) (dotted lines), and after the statistical decay of nuclear clusters (solid lines). Experimental data are taken from Ref. [24, 25].

to reproduce the saturation properties of nuclear matter, and the real part of the global Dirac optical potential. In the latest version of JAM, Coulomb and the symmetry energy potentials are implemented for the better description of lower collision energies.

The second approach recently implemented in JAM is to modify the scattering style in order to control the EoS [15-20]. In the standard implementation of the two-body scattering in the cascade type simulations, it is usually assumed that the azimuthal angle is randomly generated, while the scattering angle is selected according to the distribution which is consistent with the experimental data. Therefore, repulsive and attractive orbits are randomly selected; as a result, the two-body collision term does not contribute to the pressure in average, and the cascade simulation yields the ideal hadron resonance gas EoS in equilibrium. It has been demonstrated that the pressure of the system can be controlled by imposing the constraints [14] at each binary collision,

$$
\Delta P=\frac{\rho}{3\left(\delta \tau_{a}+\delta \tau_{b}\right)}\left(\boldsymbol{p}_{a}^{\prime}-\boldsymbol{p}_{a}\right) \cdot\left(\boldsymbol{r}_{a}-\boldsymbol{r}_{b}\right),
$$

where $\Delta P$ is the pressure generated by the two-body scattering between the particle $a$ and $b, \rho$ is the local particle density, and $\delta \tau_{a}$ is the proper time interval of the particle $a$ between successive collisions. The momentum transfer $\left(\boldsymbol{p}_{a}^{\prime}-\boldsymbol{p}_{a}\right)$ and the coordinate $\boldsymbol{r}_{a, b}$ should be evaluated in the two-body c.m. frame. We have shown that a given EoS can be simulated by choosing azimuthal scattering angle according to the constraint Eq. (4). As this approach does not require potentials, numerical cost is the same as the cascade simulations.

Finally, we have recently integrate hydrodynamical evolution into JAM dynamically [23]. In this approach, a dynamical coupling of microscopic transport model and macroscopic hydrodynamics are realized by the source terms:

$$
\begin{array}{ll}
\partial T_{p}^{\mu v}=-J^{v}+F^{v}, & \partial_{\mu} N_{p}^{\mu}=-\rho_{B} \\
\partial T_{f}^{\mu v}=J^{v}-F^{v}, & \partial_{\mu} N_{f}^{\mu}=\rho_{B},
\end{array}
$$

where $T_{p}^{\mu \nu}=\int d^{3} p p^{\mu} p^{v} / p^{0} f(x, p)$ and $T_{f}^{\mu \nu}$ are the energymomentum tensor of the particles and the fluids, respectively. Here we take the ideal fluid: $T_{f}^{\mu v}=(e+p) u^{\mu} u^{v}-$ $p g^{\mu \nu}$, where $u^{\mu}$ is the hydrodynamic four-velocity, $e$ and $p$ are the local energy density and pressure. $N_{p}^{\mu}=$ $\int d^{3} p p^{v} / p^{0} f(x, p)$ is the particle four-flow for particle system. $N_{f}^{\mu}=n u^{\mu}$ is the baryon current defined by baryon density $n$. Produced particles from the decay of strings or hadronic resonances are converted into fluid, if the local energy density at the produced point exceeds a fluidzation energy density $e_{f}=0.5-1.0 \mathrm{GeV} / \mathrm{fm}^{3}$. Thus, space-time dependent core-corona separation is incorporated in the JAM + hydro model. Particle-fluid interactions $F^{\mu}$ is not implemented: $F^{\mu}=0$ in the present version of JAM. Fluid elements are converted into particles when energy density becomes $e_{p}=0.5 \mathrm{GeV} / \mathrm{fm}^{3}$, and particles can interact with each other until the freeze-out. 

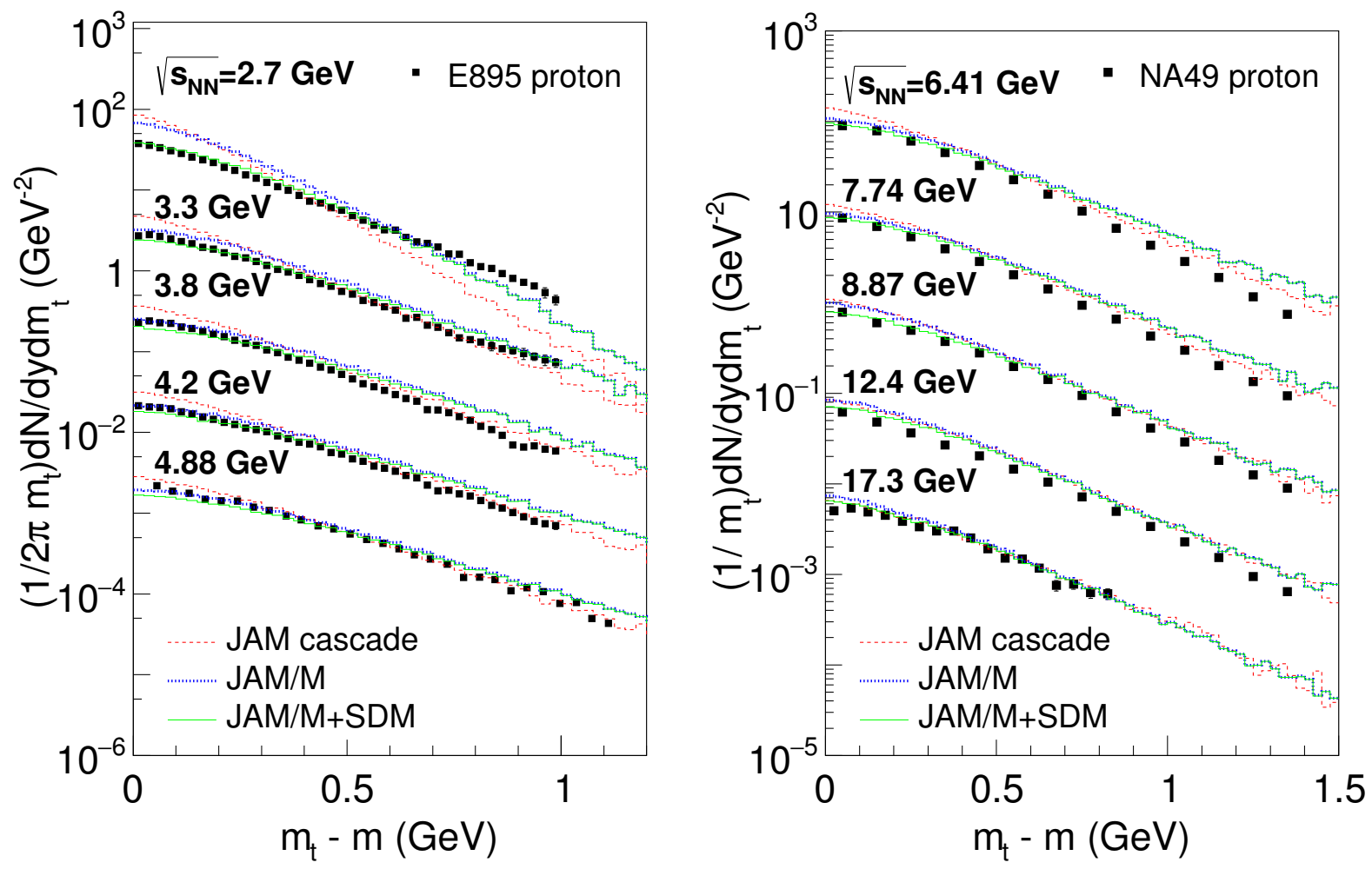

Figure 2. Transverse mass distributions for protons in central $\mathrm{Au}+\mathrm{Au}(\mathrm{Pb}+\mathrm{Pb})$ collisions at AGS (SPS) energies are compared with the JAM cascade mode (dashed lines), JAM mean-field mode (JAM/M) (dotted lines), and JAM/M + statistical decay (solid lines). Experimental data are taken from Ref. [24, 26]. The spectra are scaled down by successive factors of 10 from the 2.7 and the $6.41 \mathrm{GeV}$ data.

\section{Results}

We first examine the effects of nuclear potentials on the rapidity and the transverse mass distributions in central $\mathrm{Au}$ + Au collisions at AGS energies $\left(\sqrt{s_{N N}}=2.7-4.88 \mathrm{GeV}\right)$ and $\mathrm{Pb}+\mathrm{Pb}$ collisions at SPS energies $\left(\sqrt{s_{N N}}=6.41-\right.$ $17.3 \mathrm{GeV})$.

Figure 1 shows the rapidity distributions of protons in central $\mathrm{Au}+\mathrm{Au}$ collisions at AGS energies. It is seen that JAM with nuclear mean-field simulations predict a stronger stopping than the experimental data. A possible reason is the effect of nuclear cluster [29]. The dotted lines represent the results in which nuclear clusters are excluded, i.e. only 'free' protons are plotted. The formation of nuclear clusters is obtained by the phase space coalescence: if the relative distances and momenta between nucleons are less than $R_{0}=4.0 \mathrm{fm}$ and $P_{0}=0.3 \mathrm{GeV} / c$, these nucleons are considered to belong the same nuclear cluster. Coalescence parameters $R_{0}$ and $P_{0}$ are fixed by fitting the proton rapidity distribution at the beam energy of $\sqrt{s_{N N}}=2.7 \mathrm{GeV}$ for central $\mathrm{Au}+\mathrm{Au}$ collisions, and they are the same for other energies. Nuclear fragments obtained by the coalescence of nucleons after QMD simulation are generally in the excited states. Thus statistical decay of such excited nuclear clusters are taken into account [30]. To see the effects of the statistical decay of nuclear clusters, we plot the results after performing the statistical decay by the solid lines. We include the emissions of nuclei up to the mass number of 4 as well as gamma emission in our statistical decay model (SDM). It is seen that the statistical decay of nuclear cluster is found to be important only at the lowest AGS energy $\sqrt{s_{N N}}=2.7$ $\mathrm{GeV}$.

Figure 2 compares the transverse mass distributions for protons in central $\mathrm{Au}+\mathrm{Au}$ collisions at AGS energies from E895 [24] (left panel) and $\mathrm{Pb}+\mathrm{Pb}$ collisions at SPS energies from NA49 [26] (right panel) with the JAM results. It is seen that hadronic mean-field effects on the slope is strong up to $6.4 \mathrm{GeV}$. The important effect of the mean-field is to suppress the yields of the low transverse momentum, which improves the cascade results. It is also seen that mean-field simulations yield a harder slope than the cascade results. Exclusion of nuclear cluster has also visible effects on the transverse mass distributions up to $6.4 \mathrm{GeV}$ at low momentum region.

Let us mention the nuclear mean-field effects on the anisotropic flows. It is known that nuclear mean-field effects are large up to the AGS energies $\sqrt{s_{N N}}<6 \mathrm{GeV}$ [5]. Especially, momentum dependence of the interaction has significant impact on the anisotropic flows. We also note that mean-field effects on the cumulants of the baryon multiplicity distributions have been studied in Ref. [27, 28].

We now examine the anisotropic flows within the modified scattering style approach. The azimuthal momentum distribution of particles can be expressed as a Fourier se- 

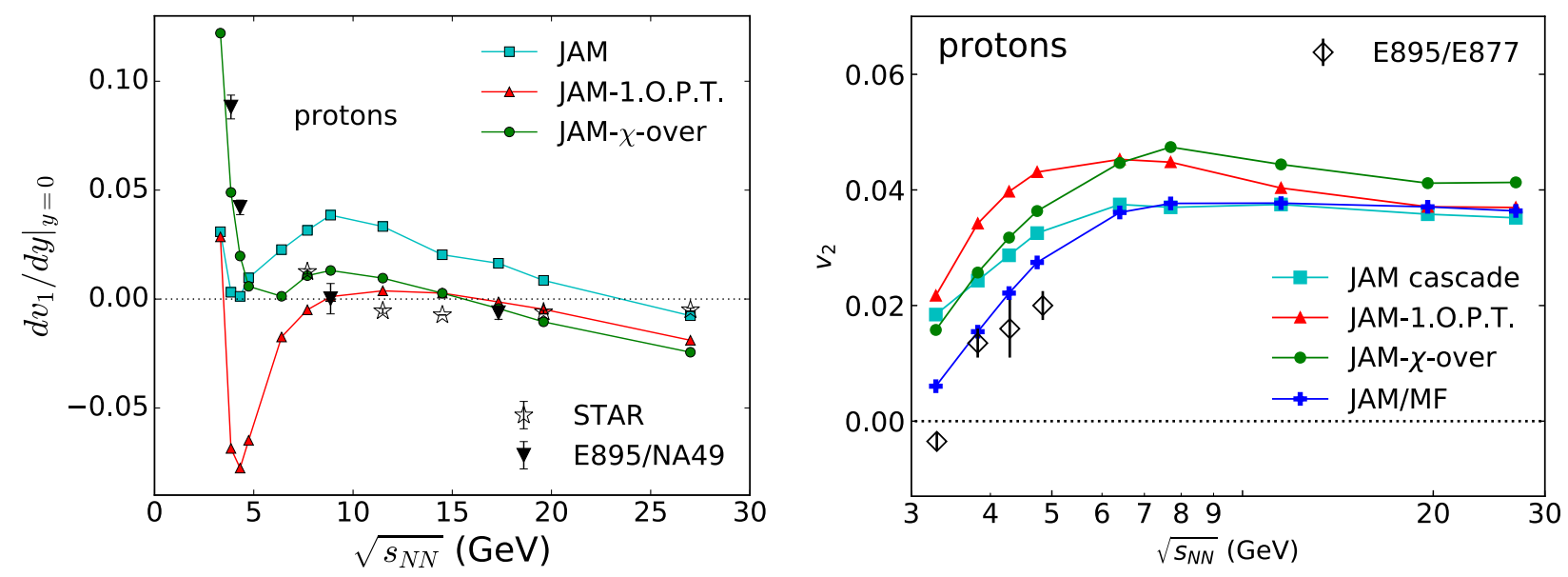

Figure 3. Beam energy dependence of the slope of $v_{1}$ and $v_{2}$ for protons in mid-central Au+Au collisions are compared with the JAM results with different EoS modes. Squares, triangles, circles, and crosses represent the results form JAM cascade mode, JAM with a first-order phase transition, JAM with crossover EoS, and JAM mean-field mode, respectively.

ries [31],

$$
E \frac{d^{3} N}{d^{3} p}=\frac{1}{2 \pi} \frac{d^{2} N}{p_{T} d p_{T} d y}\left(1+\sum_{n=1}^{\infty} 2 v_{n} \cos (n \phi)\right)
$$

where $\phi$ is the azimuthal angle with respect to the reaction plane. The coefficient $v_{1}=\langle\cos \phi\rangle$ is called directed flow, and $v_{2}=\langle\cos 2 \phi\rangle$ is called elliptic flow, where the average runs over all particles as well as all events. In the left panel of the Fig. 3, we plot the slopes of the directed flow $v_{1}$ at mid-rapidity for protons from JAM simulations as a function of the beam energy in mid-central $\mathrm{Au}+\mathrm{Au}$ collisions at $\sqrt{s_{N N}}=3-30 \mathrm{GeV}$. It is seen that the sensitivity of the EoS on the directed flow excitation function is very strong. Especially, in the case of a first-order phase transition (1.O.P.T.), there is a minimum at $\sqrt{s_{N N}} \approx 5 \mathrm{GeV}$ due to the softening of the EoS as originally predicted by the one-fluid calculations [33] as well as three-fluid models [34]. We note that the origin of the negative slope of protons at higher beam energy $\sqrt{s_{N N}}>30 \mathrm{GeV}$ is not due to softening of EoS, but is the space-momentum correlation as explained in Ref. [35]. Our model prediction with 1.O.P.T. predicts strong softening effect at $5 \mathrm{GeV}$ which is inconsistent with the experimental data. Furthermore, interestingly, STAR data exhibit the negative slope of proton directed flow from $11.5 \mathrm{GeV}$, which cannot be explained by our model. We note that other microscopic transport models such as UrQMD and PHSD [36] including the UrQMD + hydro hybird model [37] also predict positive proton slope at $11.5 \mathrm{GeV}$. Three fluid model is the only model which predicts negative $v_{1}$ slope at $11.5 \mathrm{GeV}$ [38] which also show strong sensitivities of the EoS on the directed flow. However, the UrQMD + hydro model predicts no sensitivities of EoS on the directed flow, which indicates that sizeable amount of the directed flow is generated at the early non-equilibrium stage of the collisions, where EoS effects are neglected in the UrQMD + hydro model. In the near future, a more detailed theoretical analysis of the softening effect should be addressed by employing a realistic EoS which includes non-equilibrium effects.

Elliptic flow has been considered to be a sensible probe of the EoS as well. At very high energies $\sqrt{s_{N N}}>30 \mathrm{GeV}$, strong positive elliptic flow are found which is consistent with the predictions by hydrodynamics. At lower energies $\sqrt{s_{N N}}<3 \mathrm{GeV}$, elliptic flow becomes negative due to the spectator shadowing. Thus, at the baryon stopping region $\sqrt{s_{N N}}=3-10 \mathrm{GeV}$, final strength of the elliptic flow is determined by the interplay between out-of-plane emission (squeeze-out) and in-plane emission. The excitation function of the proton elliptic flow $v_{2}$ from JAM simulations are shown in the right panel of Fig. 3. It is seen that the elliptic flow is suppressed at $\sqrt{s_{N N}}<6 \mathrm{GeV}$ by the mean-field consistent with the experimental data. The suppression of elliptic flow is due to the hard expansion of the system by the strong pressure generated by the repulsive potentials. This leads to the strong interaction with the spectator matter. Interaction with the spectator matter is the origin of the out-of-plane emission known as a spectator shadowing. In contrast, JAM with a first-order phase transition predicts an enhancement of $v_{2}$ which can be understood by the suppression of spectator shadowing due to the softening of EoS. Softening of EoS leads to slow expansion due to less pressure, and the system tends to remain in this low pressure region for a long time. Spectator shadowing effects on the directed and elliptic flows have been systematically studied in Ref. [20]. Furthermore, it is recently shown that $v_{4}$ is also enhanced by the softening [39]. An interesting feature of the enhancement of $v_{4}$ is that $v_{4}$ is positive for the out-of-plane emission in contrast to the $v_{2}$. Future measurements of the $v_{2}$ and $v_{4}$ at the beam energy region $\sqrt{s_{N N}} \approx 5-6 \mathrm{GeV}$ reveal the possible signal of the softening of the EoS.

We now turn to the JAM + hydro results. All results from the JAM + hydro model presented in this work are computed by using a EoS with a first-order phase transition [32]. A detailed study of the EoS depen- 


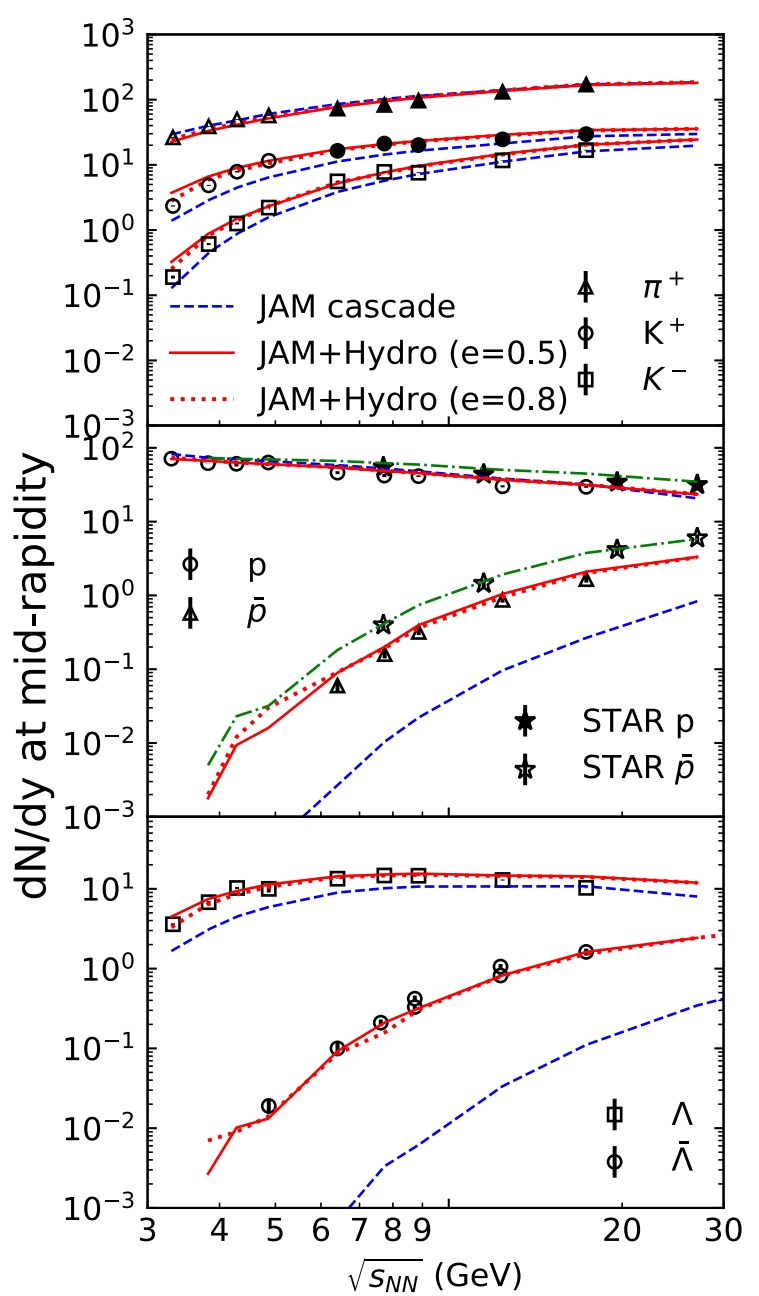

Figure 4. Comparison of the beam energy dependence of particle multiplicities at mid-rapidity in central $\mathrm{Au}+\mathrm{Au} / \mathrm{Pb}+\mathrm{Pb}$ collisions. Dashed lines represent the JAM cascade results. Solid, dotted, and dotted-dashed lines represent the calculations with the fluidzation energy density $e_{f}=0.5,0.8$, and $1.0 \mathrm{GeV} / \mathrm{fm}^{3}$, respectively. Dotted-dashed green lines correspond to the results of JAM + hydro model which contain the weak-decay feed-down contributions to (anti)-proton yield. The experimental data are taken from Refs. [24, 40-43].

dence will be presented elsewhere. In Fig. 4, JAM + hydro model calculations for particle multiplicities of pions, kaons, (anti-) protons, and (anti-) $\Lambda$ s at mid-rapidity in central $\mathrm{Au}+\mathrm{Au} / \mathrm{Pb}+\mathrm{Pb}$ collisions are compared with the data. Cascade model overestimates pion yields, and underestimates strange particle productions as well as anti-baryons. We note that both hadronic mean-field and modified scattering style have almost no influence on the strangeness and anti-baryon yields. This defect is remedied by introducing the partial-thermal equilibration of the system. JAM + hydro hybrid approach suppresses pion yields and enhances strange particles as well as anti-protons and anti$\Lambda$, and good agreement with the data is obtained.

Furthermore, beam energy dependence of the $K / \pi$ ratios from JAM + hydro model is in good agreement

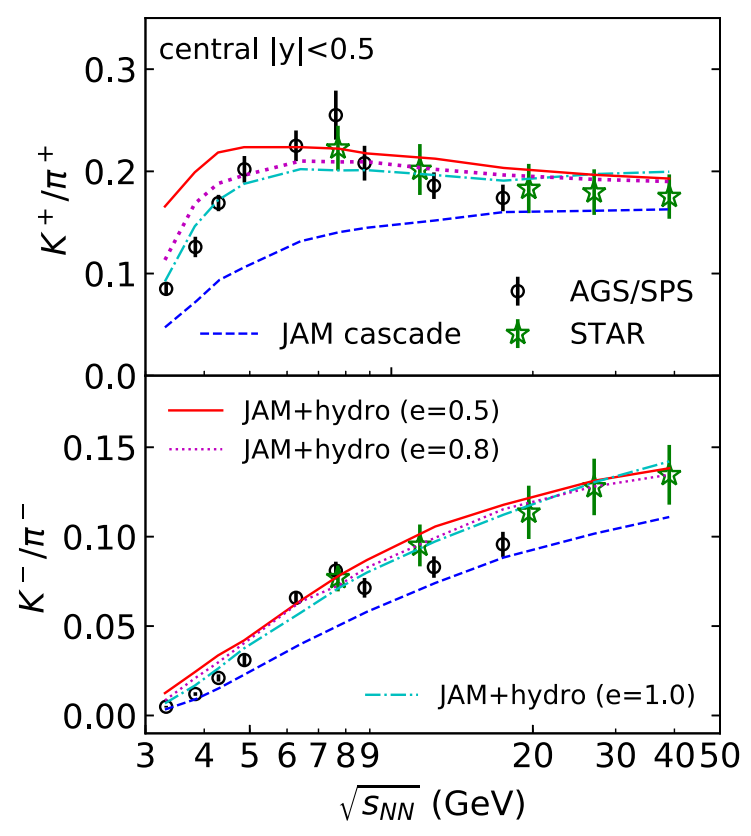

Figure 5. Comparison of the beam energy dependence of $K / \pi$ ratios at mid-rapidity in central $\mathrm{Au}+\mathrm{Au} / \mathrm{Pb}+\mathrm{Pb}$ collisions. The experimental data are taken from Refs. [44-46].

with the data for the fluidzation energy density $e_{f}=1.0$ $\mathrm{Gev} / \mathrm{fm}^{3}$. Present simulations assume the constant $e_{f}$, but it must depend on the baryon density, and one expects that $e_{f}$ should be larger for higher baryon density due to the Fermi energy of the fermions and their interactions, in contrast to the constant particlization energy density $e_{p}=0.5$ $\mathrm{GeV} / \mathrm{fm}^{3}$. It should be emphasized that core-corona separation of the fluid is important to reproduce $K / \pi$ ratios. One gets too much strangeness when all parts of the system are assumed to be thermalized [47, 48]. Finally, we remark that $K / \pi$ ratio can be reproduced by taking into account the chiral symmetry restoration at high baryon densities without assuming thermalization of the system in the PHSD transport model [49].

\section{Acknowledgement}

The author would like to thank the team of the Frankfurt Institute of Advanced Studies and J-PARC-HI theory group for the collaborations. This work was partially supported by JPSP KAKENHI Grant Number JP17K05448.

\section{References}

[1] Y. Nara, N. Otuka, A. Ohnishi, K. Niita, and S. Chiba, Phys. Rev. C 61, 024901 (2000).

[2] H. Sorge, Phys. Rev. C 52, 3291 (1995).

[3] S. A. Bass et al., Prog. Part. Nucl. Phys. 41, 255 (1998); M. Bleicher et al., J. Phys. G 25, 1859 (1999). 789,1083

[4] H. Stoecker and W. Greiner, Phys. Rept. 137, 277 (1986). 
[5] P. Danielewicz, R. Lacey and W. G. Lynch, Science 298, 1592 (2002).

[6] G. F. Bertsch, H. Kruse and S. D. Gupta, Phys. Rev. C 29, 673 (1984) Erratum: [Phys. Rev. C 33, 1107 (1986)]; H. Kruse, B. V. Jacak and H. Stoecker, Phys. Rev. Lett. 54, 289 (1985); G. F. Bertsch and S. Das Gupta, Phys. Rept. 160, 189 (1988).

[7] J. Aichelin and H. Stoecker, Phys. Lett. B 176, 14 (1986); J. Aichelin, Phys. Rept. 202, 233 (1991).

[8] M. Isse, A. Ohnishi, N. Otuka, P. K. Sahu and Y. Nara, Phys. Rev. C 72, 064908 (2005).

[9] Y. Nara and A. Ohnishi, Nucl. Phys. 956, 284 (2016).

[10] T. Maruyama, K. Niita, T. Maruyama, S. Chiba, Y. Nakahara and A. Iwamoto, Prog. Theor. Phys. 96, 263 (1996).

[11] M. Gyulassy, K. A. Frankel and H. Stoecker, Phys. Lett. B 110, 185 (1982).

[12] D. E. Kahana, D. Keane, Y. Pang, T. Schlagel and S. Wang, Phys. Rev. Lett. 74, 4404 (1995);

[13] P. Danielewicz and S. Pratt, Phys. Rev. C 53, 249 (1996).

[14] H. Sorge, Phys. Rev. Lett. 82, 2048 (1999).

[15] Y. Nara, H. Niemi, A. Ohnishi and H. Stoecker, Phys. Rev. C 94, no. 3, 034906 (2016).

[16] A. Ohnishi, Y. Nara, H. Niemi and H. Stoecker, Acta Phys. Polon. Supp. 10, 699 (2017).

[17] Y. Nara, H. Niemi, J. Steinheimer and H. Stoecker, Phys. Lett. B 769, 543 (2017).

[18] Y. Nara, H. Niemi, A. Ohnishi, J. Steinheimer, X. Luo and H. Stoecker, Eur. Phys. J. A 54, no. 2, 18 (2018).

[19] J. Chen, X. Luo, F. Liu and Y. Nara, Chin. Phys. C 42, no. 2, 024001 (2018).

[20] C. Zhang, J. Chen, X. Luo, F. Liu and Y. Nara, Phys. Rev. C 97, no. 6, 064913 (2018).

[21] M. Okai, K. Kawaguchi, Y. Tachibana, and T. Hirano, Phys. Rev. C 95, no. 5, 054914 (2017).

[22] C. Shen and B. Schenke, Phys. Rev. C 97, no. 2, 024907 (2018).

[23] Y. Akamatsu et al., Phys. Rev. C 98, no. 2, 024909 (2018).

[24] J. L. Klay et al. [E895 Collaboration], Phys. Rev. Lett. 88, 102301 (2002).

[25] B. B. Back et al., Phys. Rev. C 66, 054901 (2002).

[26] C. Alt et al. [NA49 Collaboration], Phys. Rev. C 73, 044910 (2006).

[27] S. He, X. Luo, Y. Nara, S. Esumi and N. Xu, Phys. Lett. B 762, 296 (2016).

[28] Y. Ye et al., arXiv:1808.06342 [nucl-th].
[29] Q. Li, Y. Wang, X. Wang, C. Shen and M. Bleicher, arXiv:1507.06033 [hep-ph].

[30] T. Maruyama, A. Ono, A. Ohnishi and H. Horiuchi, Prog. Theor. Phys. 87, 1367 (1992).

[31] S. Voloshin, and Y. Zhang, Z. Phys. C 70, 665 (1996); A. M. Poskanzer and S. A. Voloshin, Phys. Rev. C 58, 1671 (1998); S. A. Voloshin, A. M. Poskanzer, and R. Snellings. In Relativistic Heavy Ion Physics, pages 293 - 333. Springer, 2010. [arXiv:0809.2949].

[32] J. Sollfrank, P. Huovinen, M. Kataja, P. V. Ruuskanen, M. Prakash, and R. Venugopalan, Phys. Rev. C 55, 392 (1997).

[33] D. H. Rischke, Y. Pursun, J. A. Maruhn, H. Stoecker and W. Greiner, Heavy Ion Phys. 1, 309 (1995).

[34] J. Brachmann, S. Soff, A. Dumitru, H. Stoecker, J. A. Maruhn, W. Greiner, L. V. Bravina and D. H. Rischke, Phys. Rev. C 61, 024909 (2000).

[35] R. J. M. Snellings, H. Sorge, S. A. Voloshin, F. Q. Wang and N. Xu, Phys. Rev. Lett. 84, 2803 (2000).

[36] V. P. Konchakovski, W. Cassing, Y. B. Ivanov and V. D. Toneev, Phys. Rev. C 90, no. 1, 014903 (2014).

[37] J. Steinheimer, J. Auvinen, H. Petersen, M. Bleicher and H. Stoecker, Phys. Rev. C 89, no. 5, 054913 (2014).

[38] Y. B. Ivanov and A. A. Soldatov, Phys. Rev. C 91, no. 2, 024915 (2015); Y. B. Ivanov and A. A. Soldatov, Eur. Phys. J. A 52, no. 1, 10 (2016); P. Batyuk et al., Phys. Rev. C 94, 044917 (2016).

[39] Y. Nara, J. Steinheimer and H. Stoecker, arXiv: 1809.04237 [nucl-th].

[40] L. Ahle et al. [E866 and E917 Collaborations], Phys. Lett. B 476, 1 (2000).

[41] C. Blume, M. Gazdzicki, B. Lungwitz, M. Mitrovski, P. Seyboth, and H. Stroebele, NA49 Compilation.

[42] C. Blume and C. Markert, Prog. Part. Nucl. Phys. 66, 834 (2011).

[43] L. Ahle et al. [E-802 Collaboration], Phys. Rev. C 57, no. 2, R466 (1998).

[44] L. Adamczyk et al. [STAR Collaboration], Phys. Rev. C 96, no. 4, 044904 (2017).

[45] S. V. Afanasiev et al. [NA49 Collaboration], Phys. Rev. C 66, 054902 (2002).

[46] C. Alt et al. [NA49 Collaboration], Phys. Rev. C 77, 024903 (2008).

[47] J. Steinheimer and M. Bleicher, Phys. Rev. C 84, 024905 (2011).

[48] Y. B. Ivanov, Phys. Rev. C 87, no. 6, 064905 (2013).

[49] W. Cassing, A. Palmese, P. Moreau, and E. L. Bratkovskaya, Phys. Rev. C 93, 014902 (2016); A. Palmese, W. Cassing, E. Seifert, T. Steinert, P. Moreau, and E. L. Bratkovskaya, Phys. Rev. C 94, no. 4, 044912 (2016). 